\title{
The Effects of the Quantum and Finesse Bridles on Equine M. Brachiocephalicus and $M$. Splenius Function at Three Different Speeds
}

\author{
Emilie Gertz, Katia Gebara, Vibeke Elbrønd, Adrian Harrison \\ PAS (Physiology), Faculty of Health and Medical Sciences, University of Copenhagen, Copenhagen, Denmark \\ Email: adh@sund.ku.dk
}

How to cite this paper: Gertz, E., Gebara, K., Elbrønd, V. and Harrison, A. (2020) The Effects of the Quantum and Finesse Bridles on Equine M. Brachiocephalicus and M. Splenius Function at Three Different Speeds. Open Journal of Veterinary Medicine, 10, 65-79.

https://doi.org/10.4236/ojvm.2020.106006

Received: April 5, 2020

Accepted: June 13, 2020

Published: June 16, 2020

Copyright $\odot 2020$ by author(s) and Scientific Research Publishing Inc. This work is licensed under the Creative Commons Attribution International License (CC BY 4.0).

http://creativecommons.org/licenses/by/4.0/

\begin{abstract}
Badly designed and fitted tack induces physiological stress responses in horses, and may compromise animal welfare. Moreover, horses, just like humans, comprise a series of interconnected myofascial lines. However, to date there are no measurements of the effects of horse tack, such as bridles on muscle parameters. This study used acoustic myography to test whether two commercial anatomically designed and fitted bridles, have a measurable and positive effect on both equine muscle-function and performance. A Quantum bridle was tested on 12 Icelandic horses, whilst a Finesse bridle was tested on 8 Icelandic horses, and results compared with data from a standard bridle ( $\mathrm{n}$ $=12$ ) tested at the same time and under identical conditions. Sensors were placed on M. Brachiocephalicus and M. Splenius, and the horses exercised following a set protocol at three speeds. The results revealed statistically significant improvements in muscle performance as assessed by both the regularity of the recording at each speed, and the number of transient powerful contractions (spikes) for the anatomically designed and fitted bridles, compared with the standard bridle. It is concluded that the effect of anatomically designed bridles can not only be measured in the neck muscles of exercising horses, but that they appear to have a positive effect on muscle performance.
\end{abstract}

\section{Keywords}

Horse, Exercise, Myofascial Lines, Welfare, Icelandic, Anatomical Bridle

\section{Introduction}

Whether horses are performing as top-level athletes in Olympic events or as part of more local amateur competitions, their comfort should be of paramount importance. Indeed, there is now considerable focus on equine welfare and its rela- 
tion to performance, with particular emphasis on optimizing the fit of equestrian equipment [1] [2] as outlined by the Federation Equestre Internationale (FEI) Code of Conduct for the Welfare of the Horse [3].

Bridles for example are used to control horses through the application of pressure to sensitive areas of the horse's head, and then the removal of this pressure after correction has been achieved. However, if this regulatory pressure is not effectively removed, but instead remains as a result of bridle design, then adverse effects may result [4]. Bridles used in the Olympic disciplines (eventing, dressage and show-jumping) incorporate a so-called Swedish cavesson noseband, enabling greater tension but at the same time less force than with a plain cavesson [5] in [7], [6] in [7].

In addition, with regard to the headgear used to control a horse, special attention should be made to the way a bridle fits comfortably around the ears, across the forehead and poll as well as with regard to the cheek bones and the TMJ (temporomandibular joint). It is also essential that the bridle ensures that the bit is centrally placed within the horse's mouth [1] [2].

In recent years attention has been given to the design of bridles that take all of these factors into account, so-called anatomically comfortable bridles. Some of these new bridles are being marketed commercially with claims that they can improve performance, for example through reduced pressure at the headpiece, yet often these claims remain unproven and undocumented. Indeed, there is a general lack of scientific data on the sensitivity of equine tissues, such as muscles, to pressure, the changes ill-fitting equipment may have and the long-term potential for tissue damage [7] [8].

This trial has therefore been undertaken in an attempt to document and quantify the claims that custom made, and anatomically fitted bridles, have a measurable and positive effect on the muscle function and muscle performance of horses. The hypothesis tested was that custom made anatomically fitted bridles would result in both fewer muscle spikes (signs of pressure discomfort) and more regular muscle performance patterns during exercise, compared to a standard bridle.

\section{Materials and Methods}

\subsection{Animals}

A randomized controlled crossover study with 12 Icelandic horses between the ages of 5 - 17 years was adopted. Horses were randomly assigned to start the trial with either a standard bridle, or one that was custom made to improve anatomical fit and comfort; either a Quantum or a Finesse bridle (Quantum Bridle, Brookland, UK; Finesse Bridle,

https://www.equispecially.online/copy-of-finesse-bridles, DK). In order to minimize the number of confounding variables, the horses included and measured in this study were not only all of the same breed, but were also housed together and trained over the same terrain, often with the same rider. All the horses were 
familiar with those handling them and the test environment was as constant as possible for an outdoor study. To participate the horses had to be fit and well and not suffering from any known issues such as fatigue or lameness. The horses were assessed by a qualified Veterinarian (VSE) and two Veterinary students (KG \& ESG) before their final inclusion in this study. The owners of the horses were informed of the trial design and gave their full consent. All the horses in this trial were exposed to each of the three bridles and were ridden over a period of two days using the same riding pattern, and by the same rider. For more details regarding the horses that participated in this study see Table 1.

\subsection{Equipment}

Two saddles of the same brand (Park Hall - Icelandic Special, width XW; Park Hall Saddlery, Cambridge, UK: https://parkhallsaddles.com/saddles/), were used and fitted for each horse, with a gel pad if needed. The horses were fitted with a cotton saddle pad as the layer closest to the skin. Two girths of the same kind measuring $45 \mathrm{~cm}$ were used (Stübben $\mathrm{GmbH} \&$ Co., KG, DE;

https://stuebben.com/equi-soft-sattelgurt-inkl-polster.html). To ensure the correct fit of the bridles and bits, help was sought from Jill Hick, Gillian Batt and Helle Dueholm, who were present for the trial. A double-jointed snaffle was attached to both bridles (Herm. Sprenger Metallwarenfabrik GmbH, Iserlohn, Germany). The bit was placed centrally in the horse's mouth, between the canine tooth and the first premolar teeth, and tightened just enough so as to touch the commisures of the lips without rein tension.

Table 1. The details concerning those Icelandic horses that took part in this study. For further details about the study design and the inclusion criteria see the materials \& methods.

\begin{tabular}{cccc}
\hline Horse No. & Name & Gender & Age (years) \\
\hline 1 & Sokki & Gelding & 9 \\
2 & Ari & Gelding & 17 \\
3 & Kolfaxi & Gelding & 8 \\
4 & Karl & Gelding & 6 \\
5 & Isak & Gelding & 4 \\
6 & Hektor & Gelding & 9 \\
7 & Alfur & Gelding & 15 \\
8 & Keilir & Gelding & 16 \\
9 & Lodi & Gelding & 6 \\
10 & Roskva & Mare & 11 \\
11 & Saga & Mare & 12 \\
12 & Kastania & Mare & 7 \\
Mean Age & & & 10 years F \\
& & & 10 years M \\
\hline Sex & & $25 \% \mathrm{~F}$ & \\
\hline
\end{tabular}


The standard bridle

The standard bridle consisted of two parted headpieces. A neckpiece with a standard browband holding the bit into place and a cavesson noseband (see Figure 1).

The Quantum bridle

The Quantum bridle is a unique ergonomic headpiece with interchangeable components designed to give a horse the maximum freedom of movement of its head and neck when in place. It is adjustable in many ways, e.g. noseband, browband, cheek pads etc. The headpiece is wide to allow a greater surface area for pressure distribution, and it is constructed so as to avoid peak pressures. Underneath the headpiece are two soft raised pads, which serve to create a space for critical muscle attachments and ligaments on the skull. On the side of the cheek piece there are also two raised soft pads providing comfort and clearance. The Quantum bridle has rounded cheek pieces, following the natural jaw line to avoid direct pressure on the Temporal Mandibula Joint (TMJ) and enabling it to stand clear of the $\mathrm{N}$. facialis area. The chin and jaw pads are positioned so as to prevent restriction of breathing and swallowing. To allow movement of the jaw the buckles on the chin and jaw pads have an elastic element built in to their design. Finally, the overall Quantum bridle design ensures that the bit is held in an optimal and improved position, one that is thought to give more stability and room for the horse to swallow and relax its jaw

(https://www.quantumbridle.com) (see Figure 1).

The Finesse bridle

The Finesse bridle has been designed to reduce poll pressure using a special padding underneath the head piece

(https://www.equispecially.online/finessebridles). The special neck padding is developed to ensure a better blood flow when applying pressure. The padding itself is a fiber material which was first used with humans in hospitals to reduce the risk of decubitus (bed sores) in patients. The neck piece is designed to fit every horse anatomically since the fiber material from which it is made, is self-adjusting to the horse when tightened. The cheekpieces and chin piece are shaped in such a way as to avoid direct pressure on the cheeks and chin, whilst

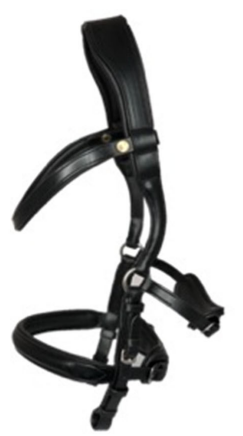

Quantum

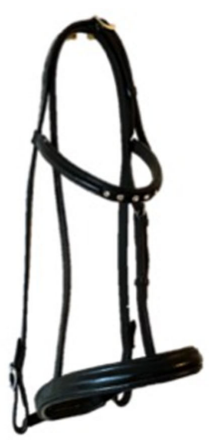

Standard

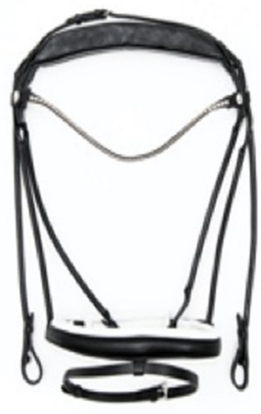

Finesse

Figure 1. The Quantum, Standard and Finesse bridles used in this study. 
the noseband is designed to be wider than that of most bridles so as to better distribute pressure. The noseband comes in different types such as cavesson with or without flash and a drop noseband. The browband is attachable and comes in various sizes fitting the individual horse. The bridle is also cushioned throughout with a soft leather padding

(https://www.equispecially.online/copy-of-finesse-bridles) (see Figure 1).

\subsection{Selected Muscles}

M. brachiocephalicus and m. splenius were the muscles chosen for measurements in this particular study. They were selected for both anatomical and functional reasons. The two muscles are part of the larger muscle groups placed dorsally and latero-ventrally on the cervical region of the spine. They are responsible for head and neck movement and $\mathrm{m}$. brachiocephalicus is also responsible for protraction of the front limb [9]. Additionally, they are involved in the equine myofascial kinetic lines [10]. Both of them are integrated in the lateral line (LL), which is split in two: 1) the superficial part, which includes the $\mathrm{m}$. brachiocephalicus, connecting in a caudal direction to the $\mathrm{m}$. cutaneus trunci, a muscle found to be involved in stabilizing the trunk [11] [12] and 2) the profound part, which includes the m. splenius, continuing into the intercostal and oblique abdominal muscles. Two other lines include these muscles, namely, 1) the front limb protraction line ( $\mathrm{m}$. brachiocephalicus), and 2) the spiral line ( $\mathrm{m}$. splenius). In the equine cranial and cervical region (head and neck) the measured muscles play an important role in terms of balancing the region, particularly with regard to the positioning of the neck, head and trunk, as well as fine-tuned balance between the neck muscles [13].

\subsection{Acoustic Myography Measurements}

Acoustic Myography (AMG-CURO-Diagnostics ApS, Bagsværd, Denmark) [14] [15] was used to measure the effects of the three bridles on the left and right sides of each horse for both $\mathrm{m}$. brachiocephalicus and $\mathrm{m}$. splenius. The horses were measured over three consecutive tests with the same rider, the same bit, saddle, reins and riding pattern, as well as under the same conditions over a period of 2 days. The riding pattern used included measurements of three different speeds; walk (slow speed), trot/tölt (medium speed) and a fast canter (fast speed) with a stop of a few seconds between each speed. The measurements from both muscles for each bridle and speed were analyzed using CURO-diagnostics ApS Software in terms of signal regularity throughout each speed and the number of spontaneous spikes that occurred (see Figure $2 \&$ Figure 3).

In brief, $50 \mathrm{~mm}$ AMG sensors were positioned on the horses over the muscles of interest. The fur between the sensor and skin was not cut, instead acoustic gel (Ekkomarine Medico A/S, Holstebro, Denmark) was placed between the skin and the sensors. The sensors were then fastened in place using adhesive bandage Snøgg (Snøgg AS, Kristiansand, Norway) and the sensors were subsequently connected to the CURO device. The muscle signal was transmitted via Wi-Fi 


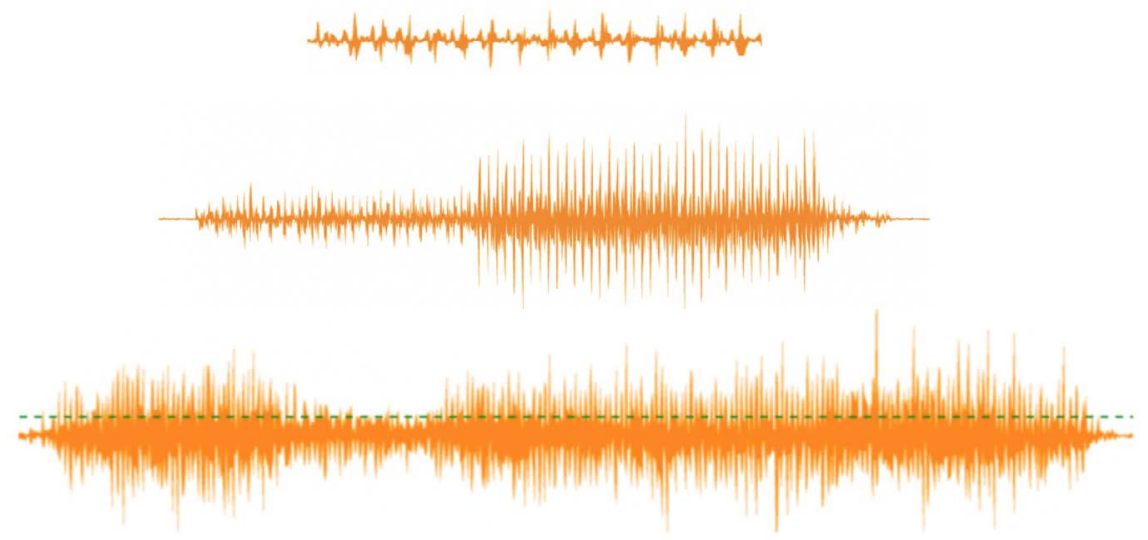

Figure 2. Examples of regular AMG signals (top two) and an irregular signal (bottom one). The one in the middle also shows a transition from one gait to another.

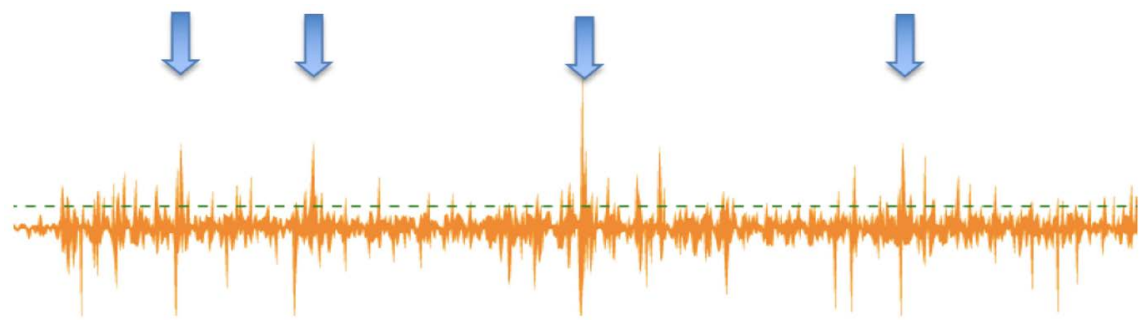

Figure 3. An example of a typical measurement with spikes highlighted with blue arrows.

from the CURO-device to an i-Pad 2 (Apple Inc, California, USA) and measurements were followed in real-time to ensure proper transmission of recordings [15] [16].

\subsection{Exercise Protocol}

Once the equipment had been secured on the horse, and the rider was in place, each horse was walked slowly for approx. 2 minutes from the tack site just outside the stables, to an outdoor arena where a set exercise protocol was rigorously followed and the different exercises and stops were marked with cones.

The exercise protocol adopted for this trial was as follows. The first exercise was a serpentine with two arches ridden in walk and then a stop. The second exercise was ridden at medium speed, either trot or tölt, one full round of the arena then a long diagonal thus changing the reins followed by one full round of the arena on the other hand, then a stop. The last exercise involved a change to the fast speed, again one full round of the arena at fast canter or pace if the horse felt like it, then a full stop. The equipage was turned around so as to change the reins before one full round of the arena at fast speed, followed by a full stop.

The protocol was designed to allow the horse to balance itself in the best way possible by not demanding high level dressage exercises. Since not all the horses were at the same level of training this protocol was also a way of accommodating for the individual horse's abilities. The protocol ensured that all the horses walked in a serpentine so as to get the best possible chance of measuring wider 
turns of both sides of the neck. During the medium and fast speeds, the rider was told to let the horse choose which gait it preferred. The rider's job was to help the horse find its own balance by just supporting the reins generously and following the horse in the saddle. The rider was also told not to force either of the horses into collection or flexion of the neck during the exercise protocol.

\subsection{Statistical Analysis}

The data were initially tested for a normal distribution. Differences between means were tested for statistical significance using GraphPad InStat 3 for Mac (Version 3.0b, 2003; GraphPad Inc., La Jolla, CA). Differences between means were tested for statistical significance using a $t$-test. Differences between means with a P-value $<0.05$ were considered significant.

\section{Results}

The horses responded well to the equipment and bridles and the results of this trial can be found in graph format below (see Figure 4 \& Figure 5). At each

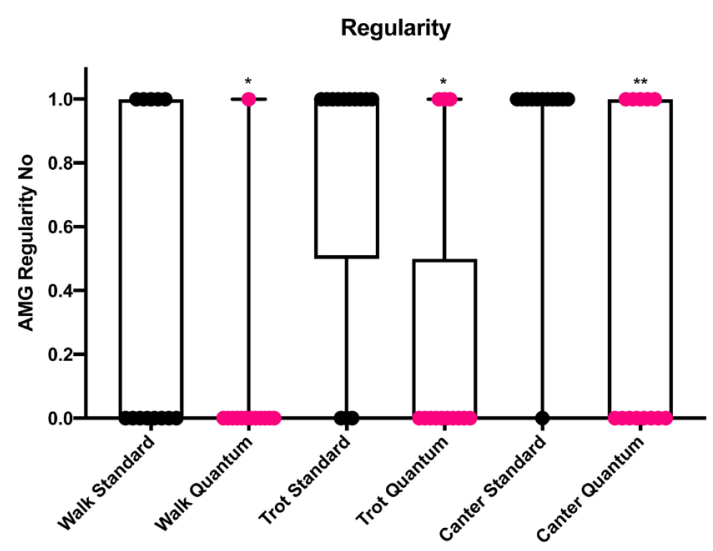

(a)

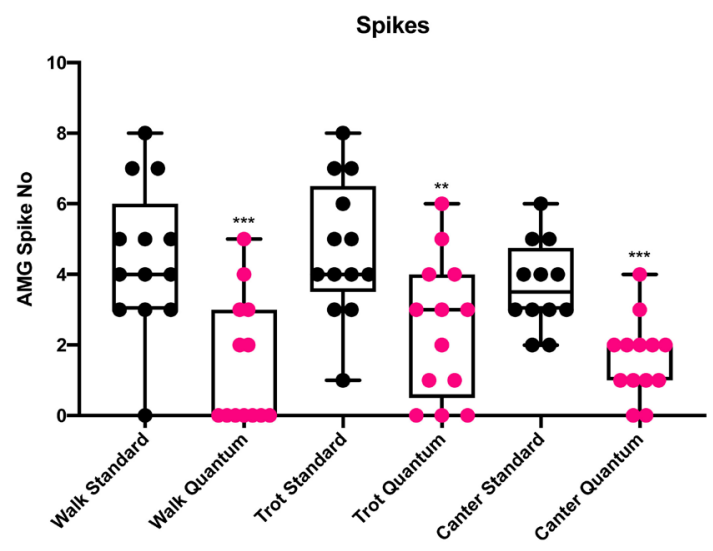

(b)

Figure 4. The combined regularity (a) and spike (b) data for both $\mathrm{m}$. brachiocephalicus and $\mathrm{m}$. splenius comparing a standard bridle $(\mathrm{n}=13)$ with the Quantum bridle $(\mathrm{n}=13)$. *denotes statistical significance of $\mathrm{P}<0.05,{ }^{* *}$ denotes statistical significance of $\mathrm{P}<0.01$, and ${ }^{* * *}$ denotes statistical significance of $\mathrm{P}<0.001$. 


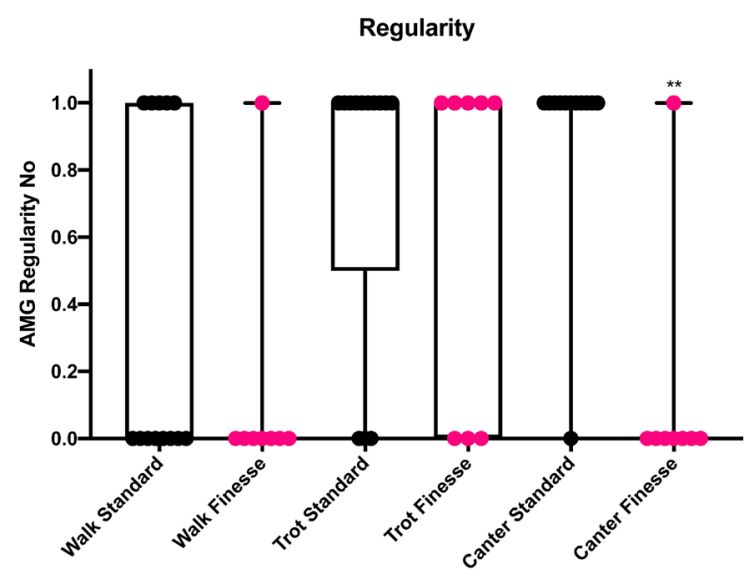

(a)

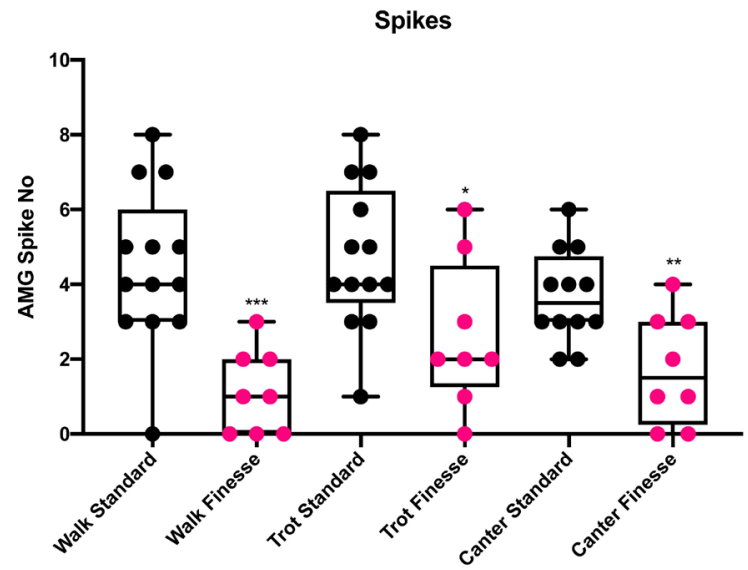

(b)

Figure 5. The combined regularity (a) and spike (b) data for both $\mathrm{m}$. brachiocephalicus and $m$. splenius comparing a standard bridle $(n=13)$ with the Finesse bridle $(n=8)$. ${ }^{*}$ denotes statistical significance of $\mathrm{P}<0.05,{ }^{* *}$ denotes statistical significance of $\mathrm{P}<0.01$, and ${ }^{* *}$ denotes statistical significance of $\mathrm{P}<0.001$.

speed the recorded AMG signals were analyzed for their regularity and assigned one of two values; 1 for irregular and 0 for regular. Figure 2 shows a typical measurement of regular muscle activity as well as a transition from one gait to another. Furthermore, the AMG signals were analyzed in terms of the number of spikes they exhibited. The spikes were defined as AMG signals of high amplitude and short duration (see Figure 3).

\subsection{Quantum Regularity and Spike Results}

The results of the regularity and spike analyses are shown for both the Quantum and Finesse bridles compared with the standard bridle (see Figure 4 \& Figure $5)$.

The combined results for both muscles for the Quantum bridle when compared to the standard bridle gave a statistically significant difference in terms of regularity for all three speeds. These changes are in favor of more regular AMG recordings for all three speeds compared with the standard bridle. 
It was likewise found that the Quantum bridle, compared to a standard bridle, resulted in significantly fewer spikes for both muscles combined at all three speeds.

\subsection{Finesse Regularity and Spike Results}

The combined results for both muscles for the Finesse bridle when compared to the standard bridle gave a statistically significant difference in terms of regularity for the canter (see Figure 5). A similar albeit non-significant improvement was also seen for the walk and trot.

It was likewise found that the Finesse bridle, compared to a standard bridle resulted in significantly fewer spikes for both muscles combined at all three speeds.

\subsection{Rider Experience}

With regard to the use of both the Quantum bridle and the Finesse bridle, the rider felt a certain positive change in the horses' ability to balance itself through the corners and turning exercises. It should be likewise noted that the rider also felt an increased willingness for the horses to speed up during medium and fast speed exercises where almost all of the horses were also easier to turn and stop using both the Quantum and Finesse bridles.

It was the rider's impression that most of the horses changed their attitude against the bit when they were fitted with the anatomically designed bridles. Some of the horses went from being unstable on the reins, fighting the bit, to accepting it and just following the reins gently, whilst other horses lowered their heads all by themselves.

Since the rider was well educated in riding Icelandic horses, they were asked to notice which gaits the horses chose in the medium and fast speed exercises. Interestingly the rider felt a positive change in the horse's choice of gait, especially for those horses that were less well trained. When changing from the standard to both of the anatomically designed bridles, the less well-trained horses went from choosing tölt at medium speed and pace at the fast speed, to preferring trot at the medium speed and canter at the fast speed.

\section{Discussion}

To the authors' knowledge, this is the first study to measure the regularity and the number or spikes in two neck muscles of horses whilst assessing the relative comfort afforded by three different bridles. It reveals that bridles that have been designed to fit with comfort have a beneficial effect on muscle function when tested using an identical exercise protocol, arena, saddle and rider, as compared to a standard bridle.

The measurements selected in this study have aimed at assessing the degree of static and dynamic pressure effects exerted through the bridle on such soft tissues as neck skeletal muscles. Recently, it was noted that equine equipment such 
as bridles, when ill-fitted or designed, may cause pain and tissue damage as a horse reacts to sudden or more long-term pressures [4] [7].

The parameter of regularity, as shown clearly in Figure 2, derives from the ability of a horse to maintain a stable muscle performance whilst performing a given activity or gait. Those horses that were not able to maintain a regular muscle signal, and constantly changed their muscle activity, were deemed to be doing so due to an unwillingness to perform or through discomfort. Every effort was made to make sure that the exercise protocol was not too strenuous or unfamiliar for the horses, and that not only was the same arena with the same saddle and rider etc. used, the change of bridle was performed randomly and in close succession to the first measurement. For this reason, we do not feel that unwillingness to perform was the major cause of the irregularity when it occurred, but rather that this was due to differences in bridle comfort.

The other parameter used in this trial, that of spikes, was assessed as per those spikes identified with a blue arrow in Figure 3, and was taken to indicate a sudden, spontaneous and voluntary muscle contraction of a powerful nature, yet of a short duration. The number of these spikes per horse was noted for both muscles during the duration of the exercise protocol, and we deem them to be indicative of signs of discomfort. They are certainly quite different in their shape and duration from those voluntary contractions observed as the result of fly irritation (AH personal observation).

This current study is not unique in terms of assessing equine physiological parameters as a result of ill-fitting tack. Fenner and colleagues [2] showed that tight fitting nosebands resulted in significant increases in not only heart rate (34 - 35 at rest to $46 \mathrm{bpm}$ ), but also eye temperature increased when compared with baseline readings, thus indicating physiological stress responses as the result of tight or badly fitted equipment on the head of horses, acting through pressure on the facial nerve and on the myofascia of the masticatory apparatus [2].

The function of $\mathrm{m}$. brachiocephalicus is lateral flexion of the head and/or extension of the shoulder joint and the elbow [9]. When contracted uni-laterally, $\mathrm{m}$. splenius draws the head to the side, and when contracted bi-laterally, it dorso-ventrally extends and raises the head and neck [9] [13]. Both of the muscles selected in this study comprise part of the lateral myofascial kinetic line (LL, [10]) and additionally part of the front limb protraction line (FLPL, m. brachiocephalicus) and the spiral line (SPL, m. splenius). Indeed, not only are the LL and SPL kinetic myofascial lines present cranially and in the neck, they both run throughout the body of the horse connecting both major muscle groups as well as the muscles in the neck to muscles in the hindlimb [10]. Where the LL is dominating in lateral flexion coupled to either flexion or extension of the trunk, the SPL is related to the diagonal coordination, cross coordination and thereby the faster speeds such as galop. The lines work together with the other body lines [10] through interconnections creating a balanced, functional and mechanical collaboration in a full body $3 \mathrm{D}$ web of myofascia. 
The data generated in this study suggest that equipment used when riding horses may potentially affect the performance of the horse through adversely applied pressure and or associated pain. It is essential that equipment such as the bridle and the saddle that sit in close contact with not only the horse's skin but also sensitive underlying anatomical structures, be measured in connection with their effect on the function of critical myofascial lines. Ultimately, improvements in this field may be achieved through the careful design of equestrian equipment that enables the distribution of pressure over a broad surface area, avoids contact with sensitive sites and uses material that is not only comfortable but also adaptable under use. Besides the equipment, it is essential that horses are trained appropriately, that is to say preventative steps be taken to ensure that weak and sensitive areas are trained to bear not only their own weight, but that of the equipment and the rider (neck/back etc). In addition, it is essential that horse's be monitored in terms of their muscle function, coordination, balance and physical endurance. With regard to improving muscle performance as a part of a larger goal towards more responsible equitation, then one should look to specific training techniques [17] [18] [19], as well as post injury manual therapies encompassing musculoskeletal physiotherapy and rehabilitation [20]. Chanda and colleagues [17] were able to show that a combination of low to moderate intensity training with bouts of high intensity training was most beneficial in terms of sports performance for Polo ponies, inducing an increase in oxidative (IIa) fibres as well as other oxidative characteristics of trained muscles [17]. In another study, resistance training, aimed at improving the performance of relatively weak fore-limb muscles, was likewise successful in two full-grown ponies trained over a period of 8 weeks, with significant improvements in muscle size and strength [18]. Yet a third study revealed that horses in an exercise programme of 30 minutes of walk-trot-canter for a period of four days a week over 14 weeks, show less muscle damage post-training compared with untrained controls [19]. Thus, exercise training lessens muscle damage as the result of physical activity and induces functional changes that can benefit both the horse and rider. Alternatively, if training is not a possibility then the use of supplements to the diet of horses has been shown to help with muscle performance, for example beta-alanine which delays the onset of neuromuscular fatigue during periods of exercise [21], although it does not improve muscle strength.

The current study has shown that two anatomically designed and comfort improved bridles have a beneficial effect on muscle physiology and function, compared with a standard bridle. The results clearly reveal that both the Quantum and the Finesse bridles resulted in significantly more regular exercise activities and far fewer spikes, taken to be transient signs of discomfort, than the standard bridle. These findings can be interpreted as indicating a lower degree of pressure on key anatomical and sensitive cranial sites in those horses fitted with the anatomically designed bridles, compared to the standard bridle. In support of the current findings, a double blinded cross-over trial of horses wearing a carefully designed bridle (bridle F) rather than the horse's usual bridle (bridle S), 
revealed that under the noseband, bridle $S$ peak pressure was $47.8 \%$ greater than that of bridle $\mathrm{F}$, and bridle $\mathrm{S}$ maximum force was $41.2 \%$ greater than that of Bridle F [8]. These authors went on to determine that bridle $\mathrm{F}$ also has a beneficial effect on the gait of the horses tested, compared with bridle $\mathrm{S}$.

The slight difference in the statistical significance in the regularity graphs of the Quantum and the Finesse bridles may be explained by the smaller sample size of the Finesse bridle data set, since there is clearly a similar trend to that shown to be significant at walk and trot for the Quantum, and the level of significance for the Quantum for both walk and trot is not that great.

The findings of this study indicate that both the anatomically designed and comfortable bridles tested have an effect through perhaps fascial tissue of the head and poll, and in an indirect way affect the two neck muscles measured. One could speculate that this effect is by means of a reduction in pressure on key sensitive anatomical sites on the head (e.g. the cheekbones) of the horse acting through the myofascial lines to alter their fine-tuned balance. It is known that there is an interconnectedness not only between muscles in the same myofascial kinetic line, but also between the myofascial lines [10], and that alterations to these myofascial lines affects muscle balance and the regularity with which they work [22]. In terms of a broader significance, say the effect of bridles per se on animal health, and looking beyond the obvious long-term consequences on horse performance and health of an imbalance to certain myofascial lines [22] [23], others have published findings with significance for this field [24] [23]. Yngvesson and colleagues [25] reported that a clinical examination of 158 tie-stall or box housed ponies in Swedish riding schools revealed an incidence of no less than 207 cases of minor skin injuries, the result of ill-fitting saddles, bridles or bits [25]. In another study, aimed at assessing the willingness of horses to accept pressure from the bit and reins through the bridle, it was found that horses would tolerate $10.2 \mathrm{~N}$ on day one of the trial but only approximately $6.0 \mathrm{~N}$ on days two and three [24]. These two findings not only show how badly fitting bridles can cause minor cuts and injuries to horses, but how quickly horses reveal conflict behaviour to tack that causes discomfort and pain.

\section{Conclusion}

It is concluded that anatomically designed bridles result in physiological measurements of neck muscle function that reveals a greater degree of regularity during periods of exercise at three different speeds, as well as fewer transient and powerful contractions, referred to in this manuscript as spikes. These findings lend weight to published data that document that ill-fitting equine tack not only results in pressure on the cranium of horses but also increases heart rate and eye temperature. The use of such anatomically designed bridles as those tested in this study appear to have a positive effect on muscle performance. 


\section{Author Contributions}

Project design was arrived at by VSE, ESG, KG and AH. Data analysis was performed by AH, ESG and KG. All authors contributed to the interpretation and writing of this manuscript.

\section{Acknowledgements}

The authors would like to thank Jill Hick and Gillian Batt for their loan of the Quantum, to Helle Dueholm for loan of the Finesse, and all the horse owners for their generous help.

\section{Conflicts of Interest}

The authors declare no conflicts of interest regarding the publication of this paper.

\section{References}

[1] Harman, J. (1999) Tack and Saddle Fit. Veterinary Clinics of North America: Equine Practice, 15, 247-261. https://doi.org/10.1016/S0749-0739(17)30175-X

[2] Fenner, K., Yoon, S., White, P., Starling, M. and McGreevy, P. (2016) The Effect of Noseband Tightening on Horses' Behavior, Eye Temperature, and Cardiac Responses. PLoS ONE, 11, e0154179. https://doi.org/10.1371/journal.pone.0154179

[3] FEI (International Equestrian Federation). Code of Conduct for the Welfare of the Horse, 2013.

https://inside.fei.org/sites/default/files/Code of Conduct Welfare Horse 1Jan2013 .pdf

[4] Uldahl, M. and Clayton, H.M. (2018) Lesions Associated with the Use of Bits, Nosebands, Spurs and Whips in Danish Competition Horses. Equine Veterinary Journal, 51, 154-162. https://doi.org/10.1111/evj.12827

[5] McGreevy, P., Warren-Smith, A. and Guisard, Y. (2012) The Effect of Double Bridles and Jaw-Clamping Crank Nosebands on Temperature of Eyes and Facial Skin of Horses. Journal of Veterinary Behavior, 7, 142-148. https://doi.org/10.1016/j.jveb.2011.08.001

[6] Casey, V., McGreevy, P., O’Muiris, E. and Doherty, O. (2013) A Preliminary Report on Estimating the Pressures Exerted by a Crank Noseband in the Horse. Journal of Veterinary Behaviour 8, 479-484. https://doi.org/10.1016/j.jveb.2013.06.003

[7] Doherty, O., Casey, V., McGreevy, P. and Arkins, S. (2017) Noseband Use in Equestrian Sports-An International Study. PLOS ONE, 12, e0169060. https://doi.org/10.1371/journal.pone.0169060

[8] Murray, R., Guire, R., Fischer, M. and Fairfax, V. (2015) A Bridle Designed to Avoid Peak Pressure Locations under the Headpiece and Noseband Is Associated with More Uniform Pressure and Increased Carpal and Tarsal Flexion, Compared with the Horse's Usual Bridle. Journal of Equine Veterinary Science, 35, 947-955. https://doi.org/10.1016/j.jevs.2015.08.023

[9] Zsoldos, R.R. and Licka, T.F. (2015) The Equine Neck and Its Function during Movement and Locomotion. Zoology, 118, 364-376. https://doi.org/10.1016/j.zool.2015.03.005

[10] Elbrønd, V.S. and Schultz, R.M. (2015) Myofascia-The Unexplored Tissue: Myo- 
fascial Kinetic Lines in Horses, a Model for Describing Locomotion Using Comparative Dissection Studies Derived from Human Lines. Medical Research Archives, 3, 1-22. https://doi.org/10.18103/mra.v0i3.125

[11] Van Iwaarden, A., Stubbs, N. and Clayton, H.M. (2012) Topographical Anatomy of the Equine M. Cutaneus Trunci in Relation to the Position of the Saddle and Girth. Journal of Equine Veterinary Science, 32, 519-524. https://doi.org/10.1016/j.jevs.2011.12.005

[12] Essig, C.M., Merritt, J.S., Stubbs, N.C. and Clayton, H.M. (2013) Localization of the Cutaneous Trunci Muscle Reflex in Horses. American Journal of Veterinary Research, 74, 1428-1432. https://doi.org/10.2460/ajvr.74.11.1428

[13] König, H.E. and Liebich, H.-G. (2014) Veterinary Anatomy of Domestic Mammals. 6th Edition, Schattauer, Stuttgart, 132-133.

[14] Harrison, A.P. (2017) A More Precise, Repeatable and Diagnostic Alternative to Surface Electromyography-An Appraisal of the Clinical Utility of Acoustic Myography. Clinical Physiology and Functional Imaging, 38, 312-325. https://doi.org/10.1111/cpf.12417

[15] Harrison, A.P., Jensen, A., Riis, K.A. and Riis-Olsen, K. (2018) Non-Invasive Assessment of Lameness in Horses with Dorsal Spinous Process Impingement "Kissing Spine": A Case Study. Multidisciplinary Advances in Veterinary Science, 1, 257-265.

[16] Andersen, N.E. and Harrison, A.P. (2019) Does Rider Weight Have a Measurable Effect on the Horse's Back Muscle Longissimus Dorsi? Integrative Journal of Veterinary Biosciences, 3, 1-4.

[17] Chanda, M., Srikuea, R., Cherdchutam, W., Chairoungdua, A. and Piyachaturawat, P. (2016) Modulating Effects of Exercise Training Regimen on Skeletal Muscle Properties in Female Polo Ponies. BMC Veterinary Research, 12, 245-252. https://doi.org/10.1186/s12917-016-0874-6

[18] Heck, R.W., McKeever, K.H., Always, S.E., Auge, W.K., Whitehead, R., Bertone, A.L. and Lombardo, J.A. (1996) Resistance Training-Induced Increases in Muscle Mass and Performance in Ponies. Medicine \& Science in Sports \& Exercise, 28, 877-883. https://doi.org/10.1097/00005768-199607000-00015

[19] White, S.H. and Warren, L.K. (2017) Submaximal Exercise Training, More than Dietary Selenium Supplementation, Improves Antioxidant Status and Ameliorates Exercise-Induced Oxidative Damage to Skeletal Muscle in Young Equine Athletes. Journal of Animal Science, 95, 657-670. https://doi.org/10.2527/jas.2016.1130

[20] Goff, L.M. (2009) Manual Therapy for the Horse-A Contemporary Perspective. Journal of Equine Veterinary Science, 29, 799-808.

https://doi.org/10.1016/j.jevs.2009.10.001

[21] Artioli, G.G., Gualano, B., Smith, A., Stout, J. and Lancha, A.H. (2010) Role of Beta-Alanine Supplementation on Muscle Carnosine and Exercise Performance. Medicine \& Science in Sports \& Exercise, 42, 1162-1173.

[22] Elbrønd, V.S., Krasnodebska, M.J. and Harrison, A. (2015) Multi-Frequency Bioimpedance and Myofascial Release Therapy: An Equine "AtlasOrange1" Validation Study. Medical Research Archives, 3, 1-14.

[23] Zügel, M., Maganaris, C.N., Wilke, J., Jurkat-Rott, K., Klingler, W., Wearing, S.C., Findley, T., Barbe, M.F., Steinacker, J.M., Vleeming, A., Bloch, W., Schleip, R. and Hodges, P.W. (2018) Fascial Tissue Research in Sports Medicine: From Molecules to Tissue Adaptation, Injury and Diagnostics: Consensus Statement. British Journal of Sports Medicine, 52, 1497-1506. https://doi.org/10.1136/bjsports-2018-099308 
[24] Christensen, J.W., Zharkikh, T.L., Antoine, A. and Malmkvist, J. (2011) Rein Tension Acceptance in Young Horses in a Voluntary Test Situation. Equine Veterinary Journal, 43, 223-228. https://doi.org/10.1111/j.2042-3306.2010.00151.x

[25] Yngvesson, J., Torres, J.C.R., Lindholm, J., Pättiniemi, A., Andersson, P. and Sassner, H. (2019) Health and Body Conditions of Riding School Horses Housed in Groups or Kept in Conventional Tie-Stall/Box Housing. Animals, 9, 73-78. https://doi.org/10.3390/ani9030073 